\title{
ARTICLE
}

\section{The aerial radiation monitoring in Japan after the Fukushima Daiichi nuclear power plant accident}

\author{
Yukihisa Sanada $^{\mathrm{a}^{*}}$, Takeshi Sugita ${ }^{\mathrm{b}}$, Yukiyasu Nishizawa ${ }^{\mathrm{a}}$, Atsuya Kondo ${ }^{\mathrm{c}}$ and Tatsuo Torii ${ }^{\mathrm{a}}$ \\ ${ }^{a}$ Headquarters of Fukushima Partnership Operations, Japan Atomic Energy Agency, 2-2-2, Uchisaiwai-cho, Chiyoda, Tokyo, \\ 100-8577, Japan; ${ }^{b}$ Science system laboratory CO. Ltd, 1342-6, Sumiyoshi, kasama-shi, Ibaraki, 309-1716, Japan; ${ }^{c}$ NESI CO. Ltd, \\ 2-1, Shiraki, Tsuruga-shi, Fukui, 919-1279, Japan
}

\begin{abstract}
The ambient dose-rate and the deposition of radioactive cesium was measured by using helicopters in the whole area of Japan to investigate the influence of the radioactivity that discharged into the atmosphere due to the disaster of the Fukushima Daiichi NPP (Nuclear Power Plant), Tokyo Electric Power Company (TEPCO), occurred by the East Japan earthquake and tsunami on March 11, 2011. As a result, the deposition of radioactive cesium on the ground was obtained, and it was clarified that range of Cs spread had been almost limited from the southern part of Iwate Prefecture to Kanto areas. The variation distribution of the dose-rate in Japan was also revealed in this measurement.
\end{abstract}

Keywords: aerial radiation monitoring; Fukushima Daiichi nuclear power plant; dose-rate; deposition of radioactive cesium; helicopter

\section{Introduction}

The Great East Japan Earthquake on March 11, 2011 generated a series of large tsunami waves that resulted serious damage to the Fukushima Daiichi nuclear power plant (NPP) and radioactive substances were discharged to the environment. To assess the scope of influence of radioactive substances diffused which spread in a wide area, the Japanese Ministry of Education, Culture, Sports, Science and Technology (MEXT) and U.S. Department of Energy (DOE) conducted joint aerial radiation monitoring (ARM) within an 80 kilometers in radius of the NPP. As a result, both the ambient dose-rate at one meter above the ground and the deposition of radioactive cesium (Cs-134, Cs-137) on the ground surface were clarified in the area $[1,2]$. To assess the affected area by the accident, we have subsequently carried out the monitoring from the surrounding area to the entire Japan. Thereby, we have obtained the distribution of the ambient dose-rate and the deposition of radioactive cesium in Japan [3].

An aerial radiation monitoring has the following advantages. (1) The widespread distribution of radionuclides can be measured in a short time with less manpower. (2) Contour maps of the deposition of the radioactive cesium can be depicted. (3) The radioactivity can be measured in the areas which people cannot come into easily, such as mountains and forests.

This paper reports an analysis performed by JAEA to

*Corresponding author. Email: sanada.yukihisa@jaea.go.jp quantify the ambient dose-rate and the deposition of radioactive cesium. In addition, its results were shown as the mapping of the whole of Japan.

\section{Aerial radiation monitoring methods}

At first, ARM was conducted in Fukushima Prefecture where the NPP is located. Then, the measurement range was expanded gradually and a flight of the whole of Japan was achieved eventually (Table 1). Measurements were taken using four helicopters. Furthermore, the ambient dose-rate was measured by portable

Table 1. Achievement of ARM.

\begin{tabular}{|c|c|c|}
\hline \multicolumn{2}{|c|}{ Date } & Area \\
\hline \multirow{6}{*}{$\stackrel{N}{\circ}$} & Apr. 6 - Apr. 29 & $\begin{array}{l}<1^{\text {st }} \text { monitoring }>60 \mathrm{~km} \text { zone from the NPP }+ \\
60-80 \mathrm{~km}^{\#}\end{array}$ \\
\hline & May 18 - May 26 & $\begin{array}{l}<2^{\text {nd }} \text { monitoring }>80-100 \mathrm{~km} \text { zone from the } \\
\text { NPP }\end{array}$ \\
\hline & May 31 - Jul. 2 & $\begin{array}{l}<3^{\text {rd }} \text { monitoring }>40 \mathrm{~km} \text { zone from the NPP }+ \\
40-80 \mathrm{~km}\end{array}$ \\
\hline & Jun. 22 - Aug. 2 & Miyagi-pref., Tochigi-pref., and Ibaraki-pref. \\
\hline & Aug. 9 - Oct. 10 & $<$ East Japan monitoring $>$ \\
\hline & Oct. 25 - Nov. 5 & $\begin{array}{l}<4^{\text {th }} \text { monitoring }>40 \mathrm{~km} \text { zone from the NPP }+ \\
40-80 \mathrm{~km} \text { zone }\end{array}$ \\
\hline \multirow{2}{*}{$\stackrel{N}{\stackrel{N}{N}}$} & Jan. 30 - May 20 & $<$ West Japan and Hokkaido monitoring > \\
\hline & Feb. 6 - Feb. 10 & $\begin{array}{l}<\text { Monitoring in the restricted area around the } \\
\text { NPP }>\end{array}$ \\
\hline
\end{tabular}


Table 2. Sensors used for Japan aerial radiation monitoring.

\begin{tabular}{cccccc}
\hline Sensor name & $\begin{array}{c}\text { Mounting } \\
\text { method }\end{array}$ & $\begin{array}{c}\text { Sensor size (in inches) and } \\
\text { amount }\end{array}$ & $\begin{array}{c}\text { Energy range } \\
(\mathrm{MeV})\end{array}$ & $\begin{array}{c}\text { No. of measurement } \\
\text { channels }\end{array}$ & $\begin{array}{c}\text { Altitude } \\
\text { measurement }\end{array}$ \\
\hline MEXT sensor & Inside & $6\left(16^{\prime \prime} \times 4^{\prime \prime} \times 2 "\right)$ & $0.02-3$ & 1,024 & GPS \\
NUSTEC sensor & Outside & $4\left(16^{\prime \prime} \times 4^{\prime \prime} \times 4^{\prime \prime}\right)$ & $0.05-3$ & 256 & Laser \\
OYO sensor & Outside & $8\left(16^{\prime \prime} \times 4^{\prime \prime} \times 4 "\right)$ & $0.2-3$ & 256 & Electric wave \\
FUGRO sensor & Inside & $4\left(16^{\prime \prime} \times 4^{\prime \prime} \times 4 "\right)$ & $0.05-3$ & 256 & GPS \\
\hline
\end{tabular}

survey-meters at 1-meter height above the ground, and the deposition of radioactive cesium was measured by Ge detectors.

By comparing the ARM data with these measuring data on the ground, we estimated the distribution of the dose-rate at a height of 1 meter above the ground and the deposition of radioactive cesium on the ground surface, mapped to determine the air dose-rate and deposition of radioactive cesium.

\subsection{Measuring system}

Large Nal detectors given in Table 2 were mounted either inside or outside the helicopters which carried 2 operators. Total counting rate (cps) and pulse height distribution data of 256 or 1024 channels were measured every second. Position and altitude of the helicopter were also measured simultaneously by a GPS sensor, and height above the ground was determined from the difference between that measurement and $90 \mathrm{~m}$ mesh digital elevation model (DEM) [4]. In case of the helicopter, height above the ground was directly measured by a laser/radio wave altimeter mounted.

In order to evaluate the dose-rate in air at a height of 1 meter from total counting rate obtained by measured altitude, a location where the helicopters could fly comparatively even in a straight line for 3 kilometers was selected as a test-line in advance. The effective attenuation coefficient $(\mu)$ of air from the ground was determined by varying altitude from 150 to $1000 \mathrm{~m}$ above the ground. Image of test-line flight is shown by Figure 1. The dose-rate conversion factor $(\mathrm{Cd}$ : cps

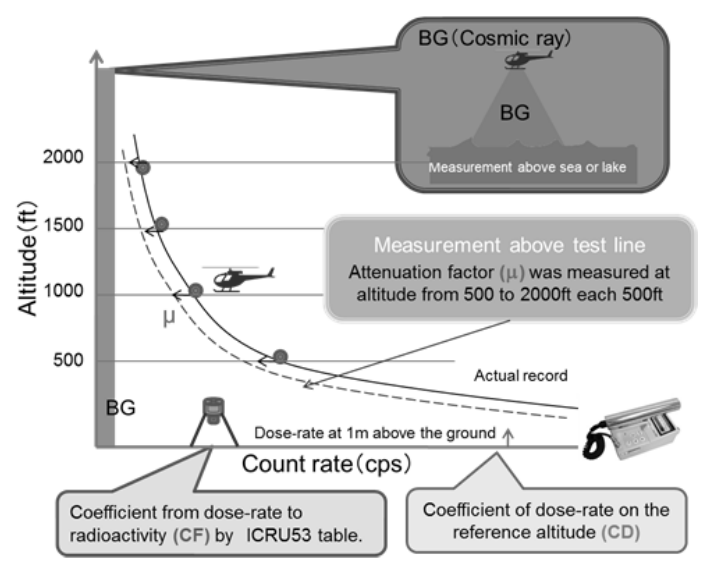

Figure 1. Image of test line flight for ARM parameters. $\left.[\mu \mathrm{Sv} / \mathrm{h}]^{-1}\right)$ is calculated by measuring the dose-rate in air directly below the test line with airborne radiation monitoring data. Also, background radiations such as cosmic rays were determined by flying about near $300 \mathrm{~m}$ above the sea, which is the standard altitude for measurement.

With actual monitoring, the helicopters flew in a comb-shaped pattern while maintaining an altitude of 150 to 300 meters above the ground. For example, if a helicopter flies at an altitude of about $300 \mathrm{~m}$ above the ground, a system will measure the average value of the radiation in a circle in $300 \mathrm{~m}$ radius on the ground. The survey line spacing within the $80 \mathrm{~km}$ range from the NPP was 1.8 to $2 \mathrm{~km}$, and measurements for other areas were taken by flying a survey line spacing of about $3 \mathrm{~km}$ or $5 \mathrm{~km}$ under ordinary circumstances. Helicopters flew at 70 - 80 knots and data sampling was carried out every second; that makes the distance between measuring points tens of meters. The flight altitude was $300 \mathrm{~m}$ above the ground.

\subsection{Analysis method of dose-rate}

Gamma-ray energy spectrum data were employed for this ARM, but counting rate in the total energy area was used for analysis for simple comparison with amount of gamma radiation on the ground. The dose-rate $\left(D_{1 \mathrm{~m}}\right.$ : $\mu \mathrm{Sv} / \mathrm{h}$ ) at the height of 1 meter from the ground is determined by the following formula using parameters ( $\mu$ and $\mathrm{Cd}$ ) which had been determined by the test-line measurement.

$$
\mathrm{D}_{1 \mathrm{~m}}[\mu \mathrm{Sv} / \mathrm{h}]=\mathrm{CR}_{\mathrm{all}} \times \frac{\exp (-\mu \cdot \mathrm{h})}{\mathrm{Cd}}
$$

where,

$\mathrm{CR}_{\text {all }}$ : the total counting rate (cps)

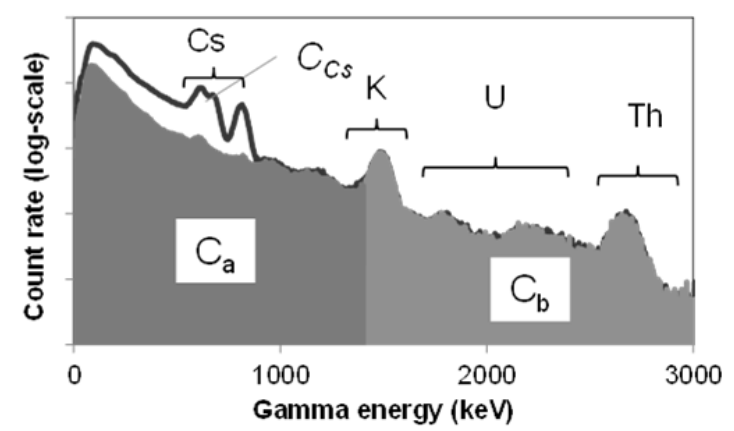

Figure 2. Image of MMGC method. 
$\mathrm{h}$ : the altitude above the ground (m).

\subsection{Method of subtracting extent of contribution of background}

The DOE has developed a Man-made Gross Counts (MMGC) method as a way to assess the concentration at low contamination areas, and uses a minimum of 1,400 $\mathrm{keV}$ as the energy range for assessing natural nuclides [5]. We attempted to adopt an improved method. Image of this method is shown in Figure 2.

The $\gamma$-rays derived from natural nuclides, such as the radon $(\mathrm{Rn})$ progenies, and cosmic rays affect the fluctuation of the background count rate in the ARM. A measured spectrum (pulse-height distribution) can be divided into two parts. One is the count rate below 1400 $\mathrm{keV}$, and the another is that above $1400 \mathrm{keV}$. From these count rate, we defined a background index (hereafter, BG-index, $\mathrm{I}_{\mathrm{BG}}$ ) as the following formula, and measured the BG-index in the area without radioactive cesium.

$$
\mathrm{I}_{\mathrm{BG}}=\left(\mathrm{C}_{\mathrm{a}}+\mathrm{C}_{\mathrm{b}}\right) / \mathrm{C}_{\mathrm{b}}
$$

where $C_{a}$ is count rate below $1400 \mathrm{keV}$ and $C_{b}$ is count rate above $1400 \mathrm{keV}$, as shown in Figure 2.

In a measured spectrum at the contaminated areas, the count rate caused by radioactive cesium $\left(\mathrm{C}_{\mathrm{Cs}}\right)$ is calculated by the following formula.

$$
\mathrm{C}_{\mathrm{Cs}}=\mathrm{C}_{\text {all }}-\left(\mathrm{C}_{>1400 \mathrm{keV}} \cdot \mathrm{I}_{\mathrm{BG}}\right)
$$

where $\mathrm{C}_{\mathrm{all}}$ is the gross count rate measured at a contaminated area, and $\mathrm{C}_{>1400 \mathrm{keV}}$ is the count rate above $1400 \mathrm{keV}$ at the same area.
The conversion factor $\left(\mathrm{CF}:\left[\mathrm{kBq} / \mathrm{m}^{2}\right][\mu \mathrm{Sv} / \mathrm{h}]^{-1}\right)$ of the deposition of radioactive cesium from the dose-rate was determined by ICRU53 table [6], and the deposition on the ground, $\mathrm{V}_{\mathrm{Cs}-134 / 137}$, was determined by subtracting background dose-rate for natural nuclide contribution at the height of 1 meter above the ground. Here, we adopted $1 \mathrm{~g} / \mathrm{cm}^{2}$ as the relaxation mass per unit area of the soil $(\beta)$.

\subsection{Mapping}

This system measure the average of the radiation emitted from the ground of $300 \mathrm{~m}$ in radius if the helicopter fly in $300 \mathrm{~m}$ above the ground. Since the line spacing of the measurement is from 1.8 to $5 \mathrm{~km}$, we must interpolate the measured result to determine the ambient dose-rate and the deposition of radioactive cesium on the ground. Therefore, an inverse distance weighted (IDW) method, whereby measurement values of 180 measurement points near the area to be analyzed are extracted and assessed as the inverse proportion of that distance, is applied as an interpolation method of the measured data.

\section{Results}

\subsection{Dose-rate and deposition of radioactive cesium}

The distribution maps of the dose-rate and the deposition of radioactive cesium obtained by this method are shown in Figure 3. As illustrated in these
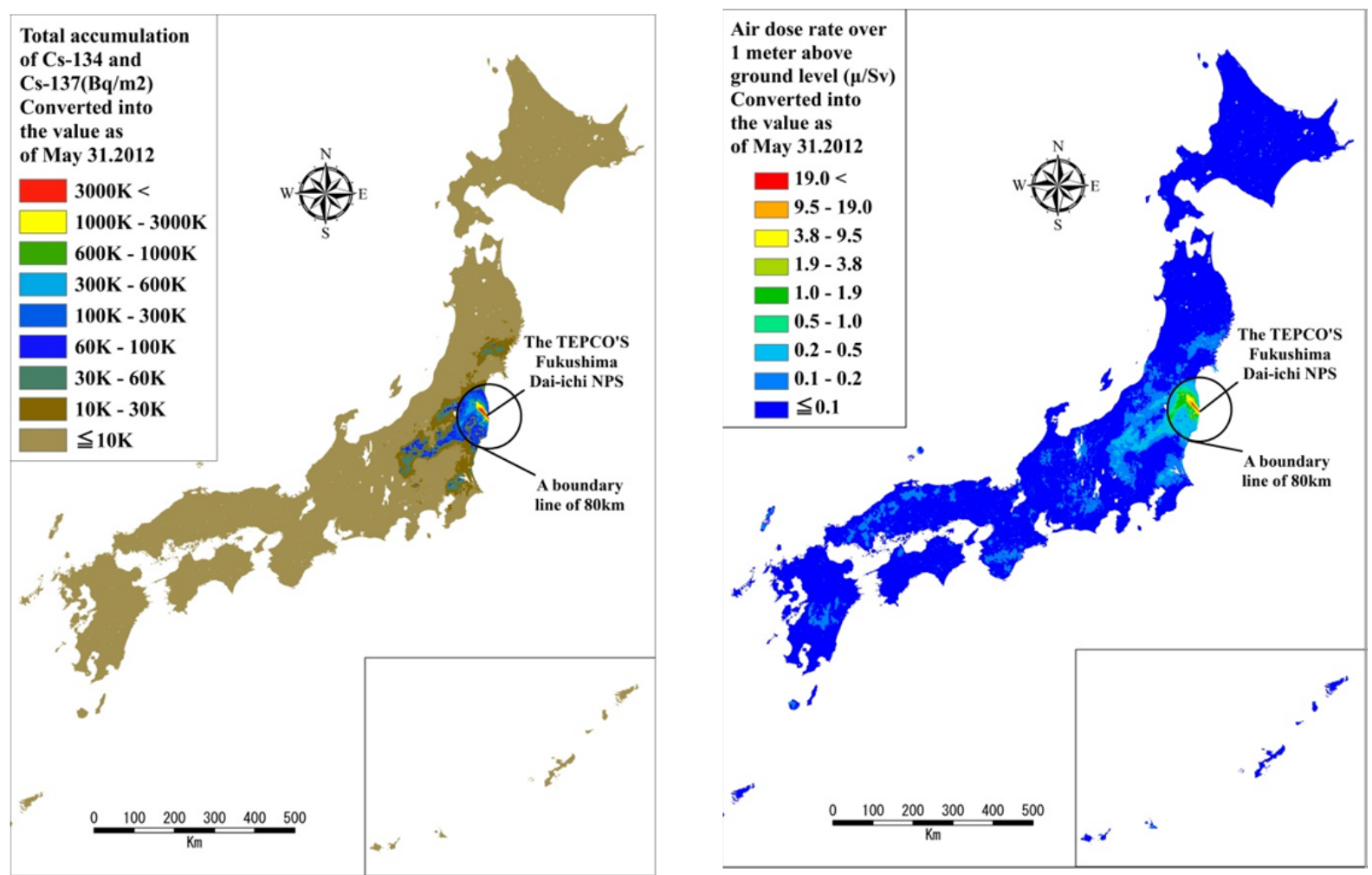

Figure 3. Map of the deposition of radioactive cesium (left) and the dose-rate at 1 meter above the ground (right) by ARM 


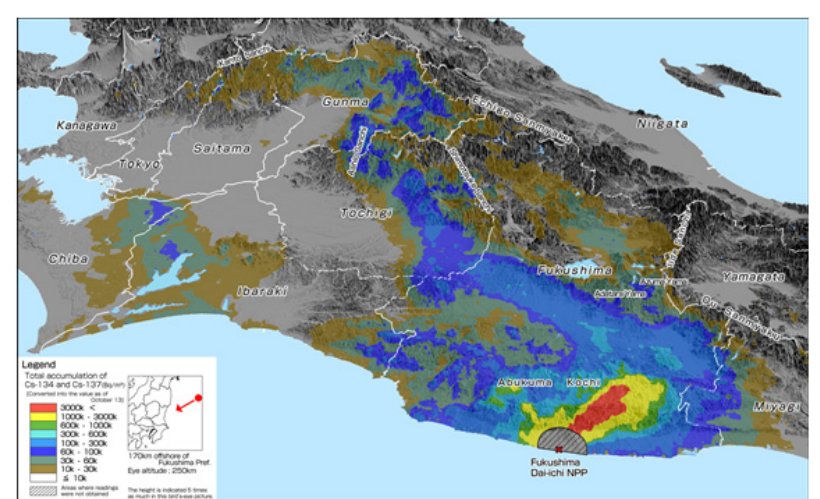

Figure 4. The 3D map of distribution of radioactive cesium.

maps, it was clarified that high dose-rate areas spread in the northwest direction from the NPP. Furthermore, the area with high dose-rate turns to southwest direction at the northeastern part of Fukushima, and reached in a part of Gunma Prefecture. In addition, the areas of the southern portions of Iwate Prefecture, Ibaraki Prefecture, and the northern portion of Chiba Prefecture were found to have a comparatively high dose-rate from 0.1 to 0.2 $\mu \mathrm{Sv} / \mathrm{h}$, caused by the deposition of radioactive cesium. The dose-rate can be seen occasionally in relatively high areas like the midland of Japan (Nagano Prefecture, Gifu Prefecture and Toyama Prefecture). These areas are consistent with the geological data contained the granite, which is involved natural nuclides such as K-40 and $\mathrm{Bi}-214$. The $\mathrm{BG}$ index is less than the standard value (average value at the areas without radioactive cesium plus its 3 sigma) in the area where the radioactive cesium wasn't detected in the spectrum in the high dose rate areas. The detection limit of the deposition of radioactive cesium was approximately $15 \mathrm{kBq} / \mathrm{m}^{2}$.

The validity of these results was evaluated by comparing with a lot of measurement results (over 4000 points) on the ground by portable survey meters [7]. However, almost all of the ground data by the survey meter measured at the flat place. ARM is carried out above the areas with rugged ground. Since main areas in Japan are mountainous areas, i.e. $70 \%$ of Fukushima Prefecture, it is necessary to examine the topographical correction in the future.

\subsection{Altitudinal distribution of the deposition of radioactive cesium}

To consider the effect of the topography at the present stage, the 3-dimensional distribution could be created for the deposition of radioactive cesium by using a GIS software. As shown in Figure 4, it was confirmed that radioactive cesium that had spread and deposited along the mountains (Ou, Iide, Echigo, Shimotsuke and Kanto Mountains) of western range from the NPP. Because almost all of the cesium deposited in areas with an elevation of less than about 1000 meters, it seems that it couldn't cross over higher mountains. On the other hand, there was the radioactive cesium in low altitude in

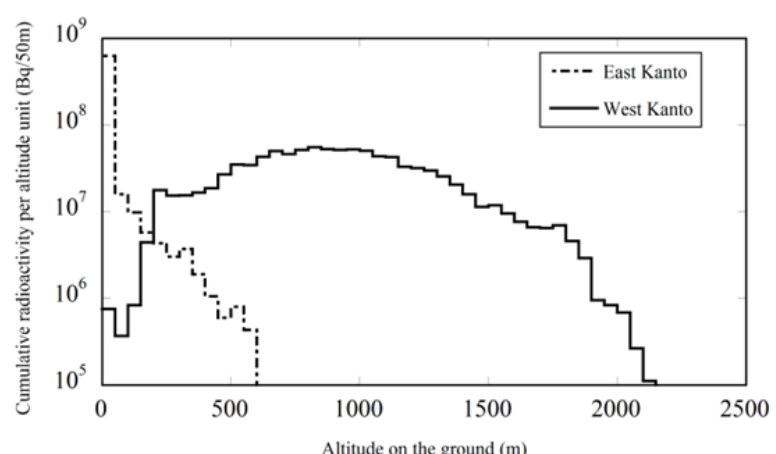

Figure 5. Altitudinal distribution of radioactive cesium western and eastern portions of Kanto.

the north area (Miyagi Prefecture) and the south area (western portion of Kanto) from the NPP. We calculated the altitude profile of the deposition of radioactive cesium every $50 \mathrm{~m}$ altitude above the ground. The example of the eastern portions (Ibaraki Prefecture) and the western portions (Gunma Prefecture) of Kanto area is shown in Figure 5. In western portion of Kanto area, radioactive cesium was widely distributed in 300 to $2000 \mathrm{~m}$, which has high mountains. On the other hand, most of the cesium had deposited at elevations of 100 meters and lower in the eastern portions of Kanto area, which have few high mountains. It can be considered that this shows that a shape of the deposition varies according to an area.

\section{Summary}

The aerial radiation monitoring of a wide range of Japan was conducted to clarify the distribution of the deposition of radioactive cesium, and we measured and analyzed the distribution of the ambient dose-rate and the deposition of radioactive cesium in various areas up to now to be confirmed. These measurements also enabled us to confirm the variation of the environmental radiation caused by natural nuclides in Japan, for which no detailed measurement results had previously existed.

\section{Acknowledgements}

This work was carried out under a contract with the MEXT in the fiscal years 2011 and 2012. More than 80 people from a lot of organizations and the enterprises participated in this monitoring project. We gratefully acknowledge for their cooperation.

\section{References}

[1] C. Lyons and D. Colton, Aerial measuring system in Japan, Health Phys. 102 (2012), pp. 509-515.

[2] D. J. Blumenthal, Introduction to the special issue on the U.S. Response to the Fukushima Accident, Health Phys. 102 (2012), pp. 482-484.

[3] Ministry of Education, Culture, Sports, Science and 
Technology (MEXT), Airborne radiation monitoring results of Japanese, (http://radioactivity.mext.go.jp/ja/monitoring_arou nd_FukushimaNPP_MEXT_DOE_airborne_monit oring/)

[4] National Aeronautics and Space Administration (NASA), (http://www2.jpl.nasa.gov/srtm/)

[5] T. J. Hendricks and S. R. Riedhauser, An Aerial
Radiological Survey of the Nevada Test Site, DOE/NV/11718-324 (1999).

[6] ICRU, Gamma-ray Spectrometry in the Environment, ICRU report 53 (1994).

[7] Y. Sanada, A. Kondo, T. Sugita and T. Torii, Distribution of radioactive cesium measured by aerial radiation monitoring, Houshasen 38 (2012), pp. 137-140. [in Japanese] 